\title{
Transaction
}

\section{Thermoplastic Polyurethane Ultrafine Fiber Web Fabricated by Laser Electrospinning}

\author{
Midori Takasaki $^{* 1}$, Kohsuke Sugihara ${ }^{* 2}$,Yutaka Ohkoshi ${ }^{* 2}$, Takayuki Fujii ${ }^{* 3}$,

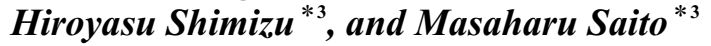 \\ ${ }^{* 1}$ Satellite Venture Business Laboratory \\ ${ }^{* 2}$ Faculty of Textile Science of Technology, Shinshu University, 3-15-1, Tokida, \\ Ueda, Nagano 386-8567, Japan \\ ${ }^{* 3}$ KB SEIREN, LTD., 6-1-1, Shimokoubata-cho, Sabae, Fukui 916-0038, Japan
}

\begin{abstract}
We have investigated the effect of the spinning conditions (the applied voltage, the laser power, the laser irradiation point, and the laser beam width) in laser-heated electrospinning, on the diameter of thermoplastic polyurethane (TPU) microfibers. The average diameter of electrospun TPU fibers decreased with decreasing applied voltage and increasing laser power. The variation in the diameters of the obtained fibers was reduced by using a narrower laser beam. A polyurethane microfiber with an average diameter of $2.4 \mu \mathrm{m}$ and a coefficient of variation of $8 \%$ was obtained using a $0.9-\mathrm{mm}$-wide laser beam. A narrower laser beam causes a steeper thinning profile across the fiber diameter and a steeper increase in the fiber temperature, which should stabilize the spinning line.
\end{abstract}

(Received 22 December, 2009 ; Accepted 19 February, 2010)

\section{Introduction}

Thermoplastic polyurethane (TPU), which has a cross-linking structure of soft and hard domains, deforms elastically and has excellent flexibility. Melt blowing is mainly used to manufacture TPU nonwovens [1-4]. Melt blowing is a simple process for forming fine fibers by blowing hot air at ultrasonic speeds, but it suffers from high variation in fiber diameter. The nonwoven obtained by the process also has poor evenness, which is detrimental to filter performance.

Recently, electrospinning has been actively investigated [5-13]. Electrospinning produces very fine fibers with submicron or nanometer-order diameters and it can be used to produce fibers from polymer solutions or molten polymers. In particular, because it is not necessary to recycle the solvent, melt electrospinning is advantageous in that it has less impact on the environment and it is suitable for industrial production processes. On the melt-electrospinning processes, laserheated electrospinning (LES), which uses $\mathrm{CO}_{2}$ laser irradiation to heat molten fibers, is excellent for producing thin, uniform fibers since it suppresses thermal degradation of fibers. This is because the fiber is drawn rapidly by electrostatic force while rapid, uniform heating is simultaneously applied [10-13]. We have applied LES to various polymers, and obtained uniform, ultrafine nylon 6 and poly (L-lactide-co- $\varepsilon$-caprolactone) fibers having average diameters of about $1 \mu \mathrm{m}$ and with a coefficient of variation (CV) of less than $20 \%$ [13].

In this present study, we investigate the effect of LES conditions (the voltage, the laser output power, the laser beam irradiation position, and the beam width) on the average diameter of ultrafine TPU fibers and its variation. We also observed the thinning position of LES for each set of spinning conditions.

In this study, we focus on the laser beam width. A laser beam has an intensity distribution across its cross section and a Gaussian distribution can generally be assumed $[15,16]$. The beam width must influence LES since a thinner laser beam heats a fiber more rapidly. Because the elongational deformation generally tends to be unstable when the material is drawn with heating [14], a shorter heating region in the spin line is preferable for fabricating uniform fibers. It is also preferable because the shorter heating region suppresses thermal degradation of the polymer. Consequently, a narrower laser beam is expected to produce thinner and more uniform ultrafine fibers.

\section{Experimental}

\subsection{Material}

TPU fibers (average diameter : $250 \mu \mathrm{m}$ ) supplied by KB Seiren Ltd. were used for spinning.

\subsection{Laser-heated electrospinning}

The LES system used in this study (Fig. 1) consists of a $\mathrm{CO}_{2}$ laser system (PIN-30R, Onizuka Glass Co., Ltd.) 
Table 1 Diameters of electrospun fibers obtained for various electrospinning conditions.

\begin{tabular}{|c|c|c|c|c|c|c|c|}
\hline \multirow[b]{2}{*}{ No. } & \multirow{2}{*}{$\begin{array}{c}\text { Laser Beam } \\
\text { Width } \\
\text { /mm }\end{array}$} & \multirow{2}{*}{$\begin{array}{c}\text { Applied } \\
\text { Voltage } \\
\text { / kV }\end{array}$} & \multirow{2}{*}{$\begin{array}{c}\text { Laser } \\
\text { Power } \\
\text { / W }\end{array}$} & \multirow[b]{2}{*}{$\begin{array}{c}d / \\
\mathrm{mm}\end{array}$} & \multicolumn{2}{|c|}{ Diameter } & \multirow[b]{2}{*}{$\begin{array}{l}\text { Uniformity of } \\
\text { obtained web }\end{array}$} \\
\hline & & & & & $\begin{array}{c}\text { Average / } \\
\mu \mathrm{m}\end{array}$ & $\begin{array}{l}\text { C.V. } \\
/ \%\end{array}$ & \\
\hline 1 & \multirow{9}{*}{2.3} & 16 & \multirow{4}{*}{6} & \multirow{4}{*}{0.5} & 2.9 & 10 & Uniform \\
\hline 2 & & 18 & & & 6.6 & 11 & Uniform \\
\hline 3 & & 20 & & & 6.7 & 12 & Uniform \\
\hline 4 & & 22 & & & 26.5 & 26 & Uniform \\
\hline 5 & & \multirow{5}{*}{18} & 6 & \multirow{3}{*}{1.5} & 3.4 & 15 & Uniform \\
\hline 6 & & & 7 & & 2.6 & 10 & Uniform \\
\hline 7 & & & 8 & & 2.3 & 11 & Uniform \\
\hline 8 & & & \multirow{2}{*}{6} & 1.0 & 4.4 & 14 & Uniform \\
\hline 9 & & & & 2.0 & 3.5 & 13 & Uniform \\
\hline 10 & \multirow{6}{*}{0.9} & & $6^{* 2}$ & \multirow{3}{*}{1.5} & 10.0 & 17 & Uniform \\
\hline 11 & & & $7^{* 2}$ & & 1.2 & 33 & Shot Observed \\
\hline 12 & & & $8^{* 2}$ & & 1.0 & 14 & Shot Observed \\
\hline 13 & & 18 & $6^{* 2}$ & \multirow{3}{*}{1.0} & 3.1 & 11 & Uniform \\
\hline 14 & & & $7^{* 2}$ & & 2.4 & 8 & Uniform \\
\hline 15 & & & $8^{* 2}$ & & 2.0 & 11 & Uniform \\
\hline
\end{tabular}

*1 coefficient of variation.

*2 Laser power measured upstream of the slit.

and an electrospinning system (NEU-010, Katotech Co., Ltd.). The TPU fiber was fed at a rate of $40 \mathrm{~mm} / \mathrm{min}$ by a feed roller. It was heated rapidly by laser irradiation (wavelength : $10.6 \mu \mathrm{m}$ ), and it was drawn by applying a high voltage between the nozzle and a copper collector plate. A TPU web was prepared by collecting the drawn fibers on the plate. The collector plate was positioned $50 \mathrm{~mm}$ from the nozzle, and 20 gauges, which have outer diameters of $0.91 \mathrm{~mm}$ and inner diameters of $0.60 \mathrm{~mm}$, were used. The relative humidity was maintained between 10 and $20 \%$ by dry air flow. Table 1 shows the other experimental parameters.

\subsection{Laser beam width measurements}

The emitted laser beam was almost parallel and was about $5 \mathrm{~mm}$ in diameter. It was deformed by a cylindrical lens, which narrows the beam in the fiber-running direction and broadens it in the perpendicular direction. In

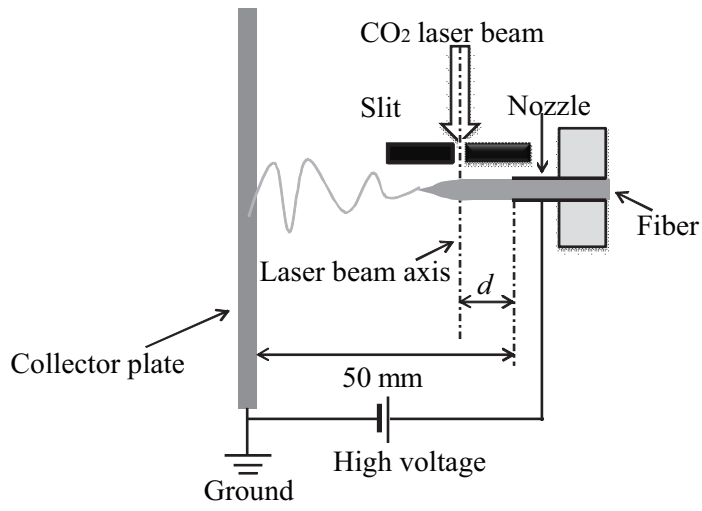

Fig. 1 Schematic diagram of laser-electrospinning system. addition, the laser beam was stopped down by an alumina slit installed before the fiber. This slit has a roundcornered rectangular aperture that is $1 \mathrm{~mm}$ wide (in the fiber-running direction) and $10 \mathrm{~mm}$ long.

The laser beam width at the irradiation point was measured with and without the slit [16]. As shown in Fig. 2, a knife edge for cutting the laser beam was installed at the fiber-running position. The output laser power was $5 \mathrm{~W}$, and the laser power was measured by a power meter after part of the laser beam was blocked by the knife edge. Each measurement was performed twice and the mean value was adopted.

Fig. 3 shows the obtained intensity profiles. The horizontal axis is the distance from the laser axis in the fiber-running direction. The total laser power was $5 \mathrm{~W}$ without the slit, and it was about $1 \mathrm{~W}$ with the slit, which indicates that the slit transmittance was about $20 \%$. Fig. 3 also shows the differential laser intensity profiles obtained

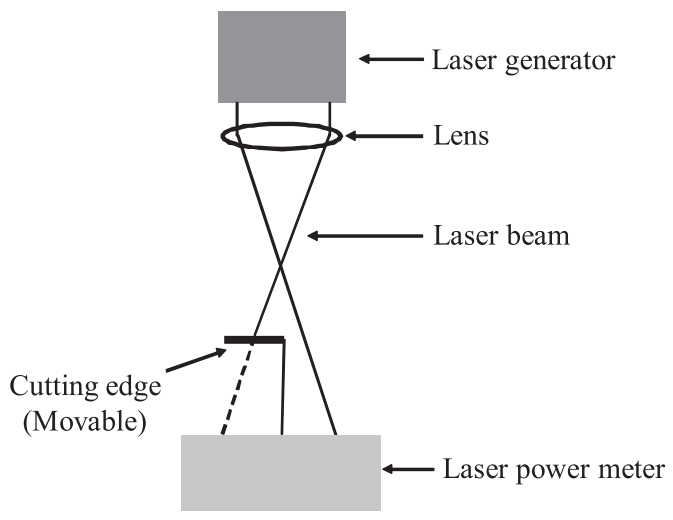

Fig. 2 Laser beam profile measurement system. 
(a)

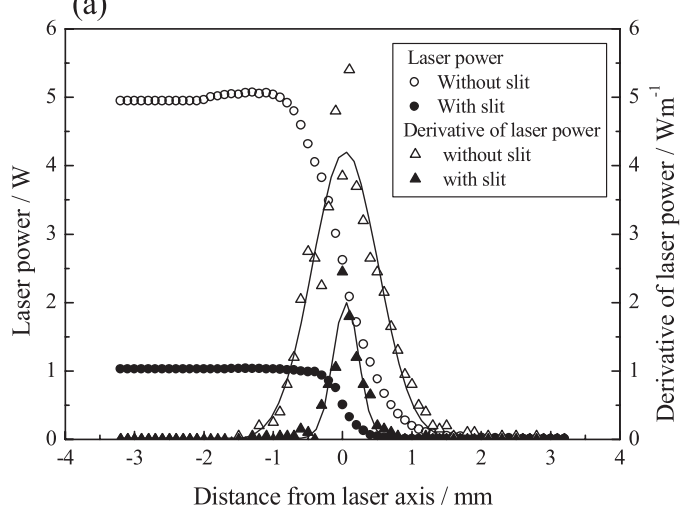

Fig. 3 Intensity profiles of laser beam obtained by measurements along the fiber axis using the system shown in Fig. 2.

using a differentiation width of $0.1 \mathrm{~mm}$ and the fitted Gaussian curves. From the width of the fitted curve, the laser beam width $\left(2 r_{\|}\right)$was estimated to be $2.3 \mathrm{~mm}$ without the slit and $0.9 \mathrm{~mm}$ with the slit. In a similar manner, we also estimated the width of the laser beam perpendicular to the fiber-running direction $\left(2 r_{\perp}\right)$ to be 7.3 with the slit and $24 \mathrm{~mm}$ without the slit. Because the $2 r_{\perp}$ values are considerably larger than the fiber diameter both with and without the slit, the laser intensity along the fiber diameter can be considered to be uniform.

\subsection{Fiber thinning observations}

Fiber thinning was recorded by a charge coupled device (CCD) (TK-C1461, Victor) equipped with a twopower telecentric lens (Optart Co. Ltd.).

\subsection{Scanning electron microscope observations}

The electrospun fibers were observed by a scanning electron microscope (SEM; S-2380N, Hitachi High Technologies Co. Ltd.). The average fiber diameter and its $\mathrm{CV}$ were calculated from over 50 diameters measured for the SEM images.

\subsection{Specific heat measurements}

The specific heat of TPU averaged for $210-240^{\circ} \mathrm{C}$ was measured by a power-compensation differential scanning calorimeter (Diamond, PerkinElmer Co. Ltd.). The TPU resin and an empty pan were held at $160^{\circ} \mathrm{C}$ for $10 \mathrm{~min}$ and were then heated to $260^{\circ} \mathrm{C}$ at a rate of $10^{\circ} \mathrm{C} / \mathrm{min}$.

\subsection{FT-IR spectroscopy}

To determine the absorption coefficient of TPU, FTIR spectra of TPU films with thicknesses of 144, 225, 278, and $316 \mu \mathrm{m}$ were measured using a FT-IR spectrometer (FT-IR 8400, Shimadzu Co. Ltd). The wavenumber resolution was $4 \mathrm{~cm}^{-1}$, the number of accumulations was 100 , and the scanning range was $500-4000 \mathrm{~cm}^{-1}$.
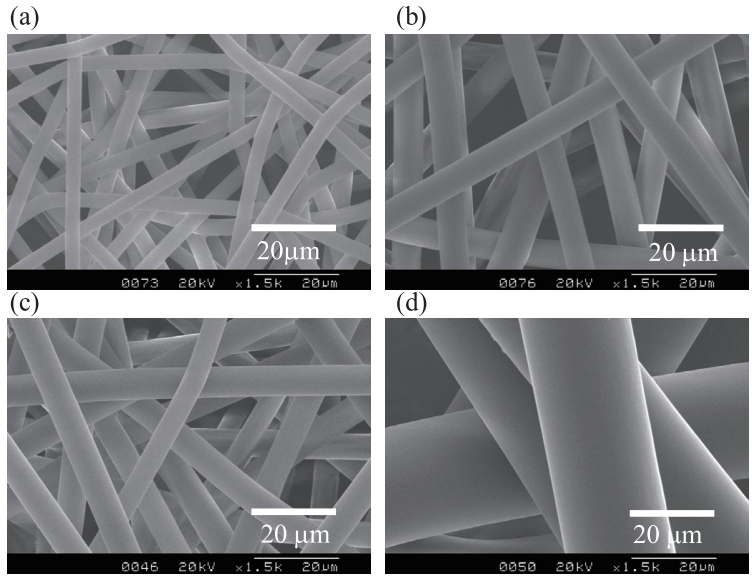

Fig. 4 SEM images of laser electrospun TPU fibers for conditions (a) No. 1, (b) No. 2, (c) No. 3, and (d) No. 4 of Table 1.

\section{Results and discussion}

\subsection{Applied voltage}

Fig. 4 shows SEM images of TPU fibers prepared with various applied voltages. Table 1 (Nos. 1-4) gives the average diameters and CVs obtained from the images. Fiber thinning was stable under all the conditions. The average fiber diameter increased with increasing applied voltage. In particular, both the average diameter and $\mathrm{CV}$ of the fiber produced using an applied voltage of $22 \mathrm{kV}$ are considerably larger than those of the other fibers.

As shown in Fig. 5, the starting point of fiber thinning for condition (d) is nearer the nozzle (i.e., further upstream) than for conditions (a)-(c). This indicates that the fiber deforms at a lower temperature (i.e., at a higher melt viscosity) because of the larger electrostatic force. It might be considered strange that a thicker fiber was obtained for a higher electrostatic force. However, this phenomenon can be explained as follows. Since the fiberrunning speed increases with thinning in the laser irradiation region, the laser energy per unit volume of the fiber decreases with increasing applied voltage. Consequently, the temperature rise and the melt viscosity drop are suppressed at higher voltages. Therefore, thicker fibers are obtained at higher applied voltages despite the electrostatic force being higher.

\subsection{Laser power}

As shown in Table 1, thinner fibers are obtained at higher laser powers. By contrast, the $\mathrm{CV}$ is almost independent of the laser power. From the thinning behavior shown in Fig. 6, the starting point of fiber thinning shifted upstream at higher laser powers. This is because a higher laser power heats the fiber more rapidly, so that the TPU fiber tends to be drawn at a lower melt viscosity further upstream where a higher electrostatic 
(a)

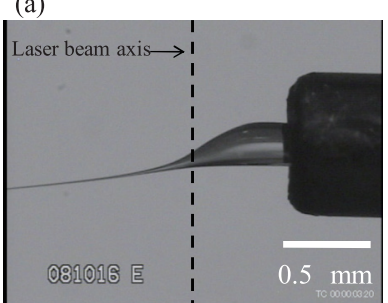

(c)

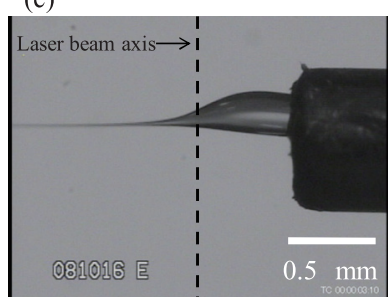

(b)

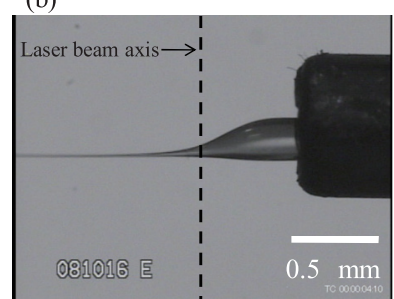

(d)

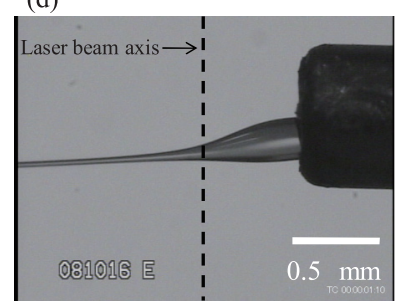

Fig. 5 Thinning behavior of TPU fibers for conditions (a) No. 1, (b) No. 2, (c) No. 3, and (d) No. 4 of Table 1.

force is applied. Therefore, thinner TPU fibers can be obtained at higher laser powers.

\subsection{Laser irradiation position}

As shown in Table 1, the fiber diameter tends to decrease with when the laser irradiation position is moved closer to the nozzle (i.e., with increasing $d$ ), with the exception of a beam width of $0.9 \mathrm{~mm}$ and a laser power of $6 \mathrm{~W}$. In addition, the fiber deformation region shifts downstream with increasing $d$, as shown in Fig. 7. Because this tendency is the same that observed when the applied voltage is reduced, it seems likely that the charge applied at the nozzle is discharged from the running fiber. Since the exceptional case (No. 10, beam width: $0.9 \mathrm{~mm}$; laser power : $6 \mathrm{~W}$ ) is for the lowest irradiated energy, it is thought to have the slowest rise in the fiber temperature and the most delayed fiber deformation. Therefore, in this case, thicker fibers were obtained at lower electrostatic forces because the discharge does not influence the irradiated power so much.

\subsection{Laser beam width}

Figs. 8 and 9 show images of fiber and spinning behavior for a beam width of $0.9 \mathrm{~mm}$ and $d=1.0$ and 1.5 $\mathrm{mm}$ in comparison with those for a beam width of $2.3 \mathrm{~mm}$ and $d=1.5 \mathrm{~mm}$. Fig. 10 shows the fiber diameter profiles obtained from the images obtained for a beam width of $0.9 \mathrm{~mm}$ and $d=1.0 \mathrm{~mm}$ and for a beam width of $2.3 \mathrm{~mm}$ and $d=1.5 \mathrm{~mm}$. At the narrower beam width $(0.9 \mathrm{~mm})$, the starting point of laser irradiation occurs about $0.7 \mathrm{~mm}$ further downstream. To compensate for this shift, we moved the laser irradiation position $0.5 \mathrm{~mm}$ upstream. The fiber thinning point moves downstream when the slit is installed and it moves upstream by reducing $d$ from 1.5 $\mathrm{mm}$ to $1.0 \mathrm{~mm}$. However, while the thinning point moves when the beam width is reduced, the laser irradiation position moves more. In addition, the elongational strain rate increases with narrowing of the beam width. In particular, the strain rates for conditions Nos. 13, 14, and 15 (which have narrower beam widths) clearly depends on the laser power.

For conditions Nos. 11 and 12 (i.e., a narrow beam width of $0.9 \mathrm{~mm}$ and $d$ was maintained at $1.5 \mathrm{~mm}$ ), a uniform fiber cannot obtained due to thermal degradation. This suggests that thermal degradation occurs when the rise in the fiber temperature is fast compared with the fiber acceleration. In contrast, a uniform fiber can be obtained for a beam width of $0.9 \mathrm{~mm}$ and $d$ of $1.0 \mathrm{~mm}$. A
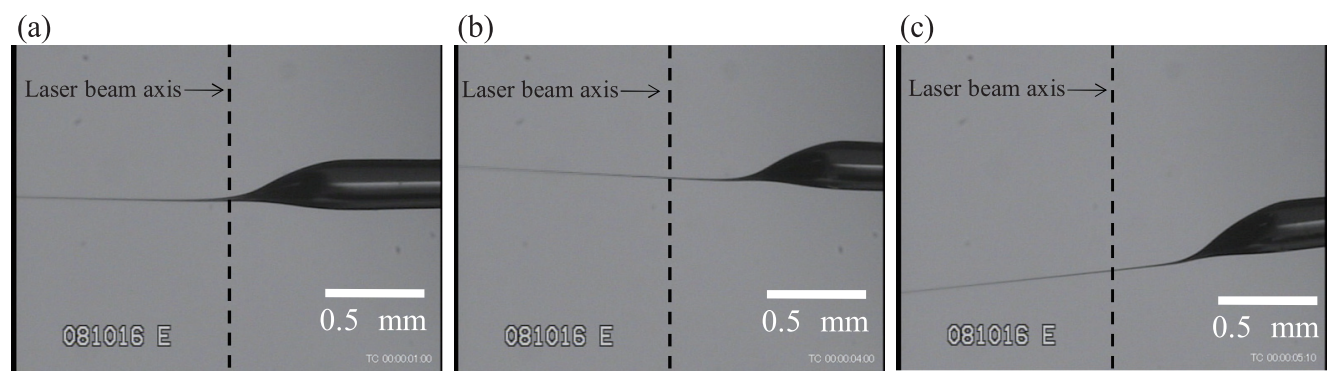

Fig. 6 Thinning behavior of TPU fibers for conditions (a) No. 5, (b) No. 6, and (c) No. 7 of Table 1.

(a)

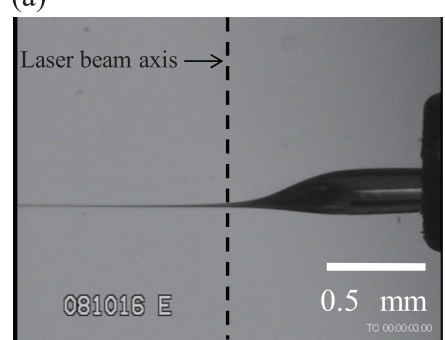

(b)

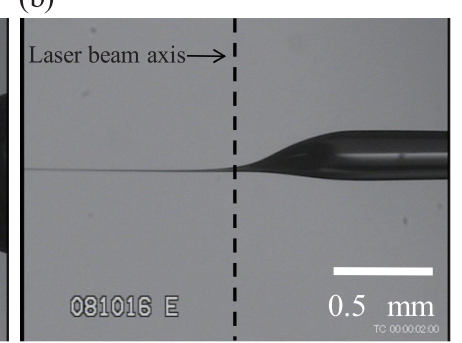

(c)

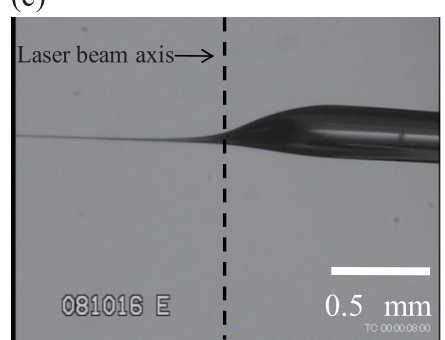

Fig. 7 Thinning behavior of TPU fibers for conditions (a) No. 8, (b) No. 5, and (c) No. 9 of Table 1. 
(a)

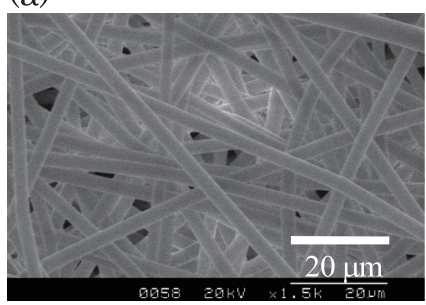

(b)

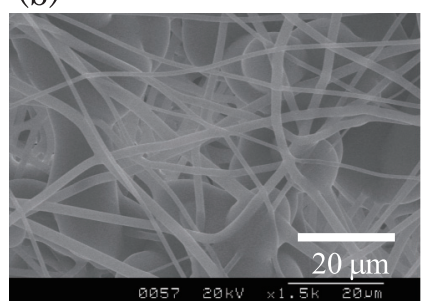

(c)

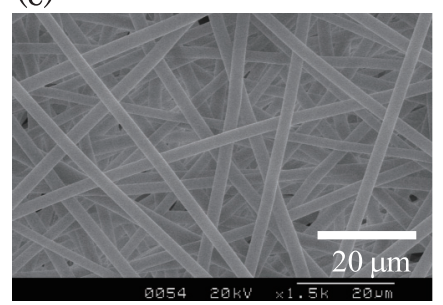

Fig. 8 SEM images of laser electrospun TPU fibers for conditions (a) No. 6, (b) No. 11, and (c) No. 14 of Table 1 .

(a)

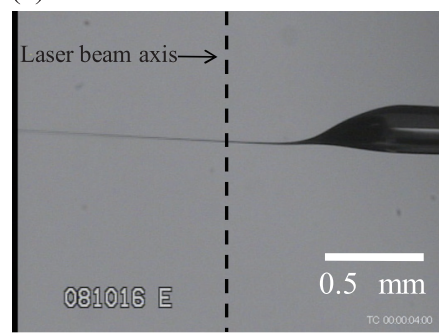

(b)

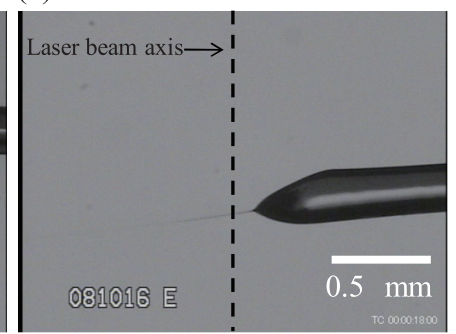

(c)

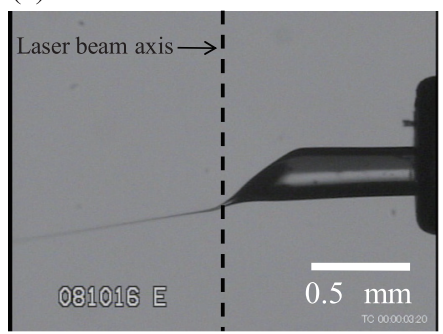

Fig. 9 Thinning behavior of TPU fibers for conditions (a) No. 6, (b) No. 11, and (c) No. 14 of Table 1.

high electrostatic force caused by the suppression of discharge seems to accelerate fiber thinning.

The fiber diameters for conditions Nos. 5, 6, and 7 are almost the same as those for conditions Nos. 13, 14, and 15. Comparing in more detail, the latter conditions (Nos. 13, 14, and 15) produce fibers with slightly thinner diameters and considerably lower $\mathrm{CV}$ values than the former conditions (Nos. 5, 6, and 7). This result confirms the explanation given above that predicts that a more uniform electrospun fiber can be obtained by narrowing the beam width. In particular, fibers obtained by conditions Nos. 14 and 15 have average diameters in the range 2.0-2.4 $\mu \mathrm{m}$ and a $\mathrm{CV}$ in the range $8-11 \%$, which indicates the variation in the fiber diameter is only about $200 \mathrm{~nm}$. These fibers are expected to be useful for producing high-efficiency filters because they can be used to produce a uniform microfiber web without using any solvent.

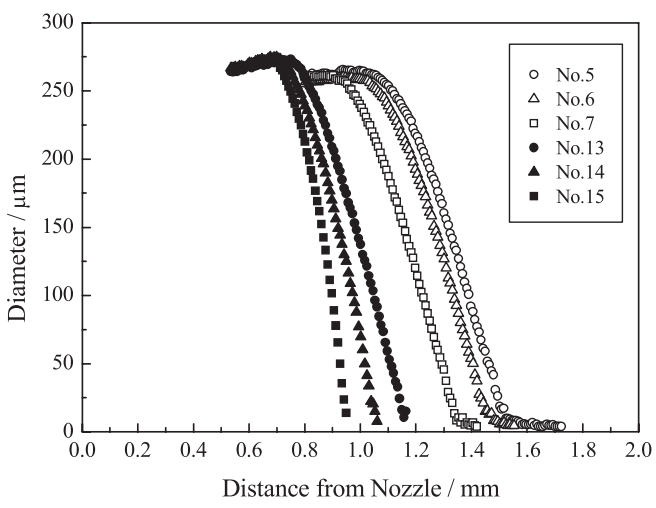

Fig. 10 Diameter profiles of TPU fibers obtained for each laser-electrospinning condition.

\subsection{Fiber temperature profile}

The fiber temperature profiles (Fig. 11) were estimated using the energy balance equation (1) $[15,16]$, where $x$ is the distance along the fiber axis, and $T$ and $T^{*}$ are the temperatures of the fiber and the atmosphere, respectively. The first and second terms on the right-hand side correspond to heating by laser irradiation and cooling by heat transfer, respectively. Heating by plastic deformation and crystallization were not considered.

$$
\frac{\Delta T}{\Delta x}=\frac{A D}{W C_{p}} \frac{2 P}{\pi r_{\|} r_{\perp}} \exp \left(-2\left(\frac{x}{r_{\|}}\right)^{2}\right)-\frac{\pi D h}{W C_{p}}\left(T-T^{*}\right)
$$

Experimental values for the total laser intensity $P$ and the laser beam width were used in the calculations. The mass flow rate $W$ was estimated from the volume flow rate and the density $\left(1.2 \mathrm{~g} / \mathrm{cm}^{3}\right)$ [17]. The absorbance of the fiber $(A=0.916)$ was calculated [15] by considering reflection from the fiber surface, assuming the fibers have circular cross sections, and taking the

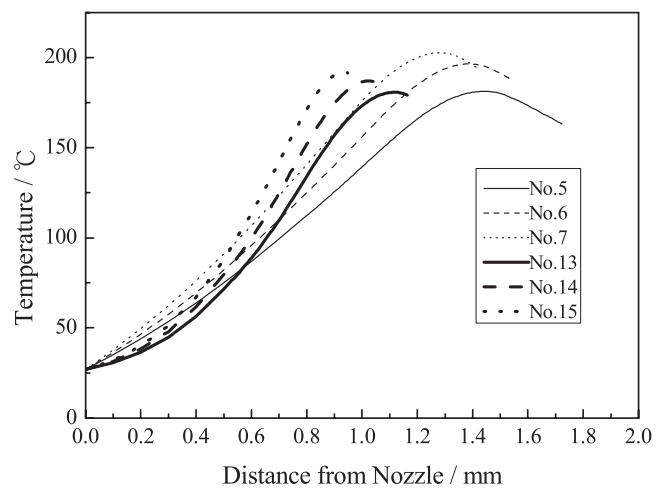

Fig. 11 Temperature profiles of TPU fibers estimated using Eq. (1). 
absorption coefficient to be $2.5 \times 10^{4} \mathrm{~m}^{-1}$ based on FT-IR $\mathrm{kg}^{-1}$ and the fiber diameter $D$ (Fig. 10) were measured, and the heat transfer $h$ was calculated using Kase and Matsuo's formula [18].

The results in Fig. 11 show that while the fiber temperature starts to rise at almost the same point for all conditions, the fiber temperature rises more steeply for a narrower laser beam width. For all conditions, the fiber deforms at temperatures in the range $160-180^{\circ} \mathrm{C}$. While slightly higher deformation temperatures were observed at higher laser powers, the deformation temperatures were almost independent of the laser beam width. The increase in the deformation rate when the laser beam width is reduced with no change in the fiber deformation temperature supports the above-mentioned assumption that the electrostatic force increases when the laser irradiation position is moved toward the nozzle. It should be possible to produce uniform, ultrafine fibers by rapidly heating a fiber subject to a high electrostatic force.

\section{Conclusions}

TPU ultrafine fiber webs were prepared by LES under various spinning conditions (applied voltage, laser power, laser beam width, and laser beam irradiation position). The effects of the spinning conditions on the fiber diameter and its variation were analyzed.

Thicker fibers were obtained at higher applied voltages and lower laser powers. The starting point of fiber thinning moved upstream when the applied voltage was increased, while it moved downstream when the laser power was reduced. In addition, moving the laser irradiation point further from the nozzle tip had the same effect as lowering the applied voltage.

By reducing the laser beam width from $2.3 \mathrm{~mm}$ to $0.9 \mathrm{~mm}$ and moving the laser irradiation point toward the nozzle, the variation in the fiber diameter was considerably reduced. As a result, a TPU web made from ultrafine, uniform fibers having an average diameter of $2.4 \mu \mathrm{m}$ and a CV of $8 \%$ could be fabricated. The temperature profiles estimated from measured diameter profiles indicate a steeper increase in the fiber temperature and more rapid fiber thinning at narrower laser beam widths. The steep increase in the fiber temperature and the rapid thinning of fiber must stabilize the spin line and reduce the variation in the diameter of the resulting fibers.

\section{Acknowledgements}

The authors express their sincere thanks to Mr. K. Morie and Mr. S. Kinugawa for helpful experimental support.

\section{References}

1. Y. E. Lee and L. C. Wadsworth, J. Appl. Polym. Sci., 105, 3723 (2007).

2. T. Zapletalova, S. Michielsen, and B. Pourdeyhimi, J. Eng. Fib. Fabr., 1, 62 (2006).

3. A. Begenir, S. Michielsen, and B. Pourdeyhimi, Polym. Eng. Sci., 49, 1340 (2009).

4. Y. Yamagawa, JPA Patent, 248454 (2000).

5. A. Formhals, US Patent, 1975504 (1934).

6. A. F. Spivak, Y. A. Dzenis, and D. H. Reneker, Mech. Res. Comm., 27, 37 (2000).

7. D. H. Reneker, A. L. Yarin, H. Fong, and S. Koombhongse, J. App. Phys., 87, 4531 (2000).

8. A. L. Yarin, S. Koombhongse, and D. H. Reneker, J. Appl. Phys., 90, 4836 (2001).

9. R. Deng, Y. Liu, Y. Ding, P. Xie, L. Luo, W. Yang, J. Appl. Polym. Sci., 114, 166 (2009).

10. N. Ogata, G. Lu, T. Iwata, S. Yamaguchi, K. Nakane, and T. Ogihara, J. Appl. Polym. Sci., 104, 1368 (2007).

11. N. Ogata, S. Yamaguchi, N. Shimada, G. Lu, T. Iwata, K. Nakane, and T. Ogihara, J. Appl. Polym. Sci., 104, 1640 (2007).

12. N. Ogata, N. Shimada, S. Yamaguchi, K. Nakane, and T. Ogihara, J. Appl. Polym. Sci., 105, 1127 (2007).

13. M.Takasaki, H. Fu, K. Nakata, Y. Ohkoshi, and T. Hirai, Sen'i Gakkaishi, 64, 29 (2008).

14. K. Toriumi, A. Konda, Sen'i Gakkaishi, 40, T-193 (1984).

15. W. Okumura, T. Yamaguchi, Y. Ohkoshi, Y. Goth, and M. Nagura, Int. Polym. Proc., 17, 125 (2002).

16. T. Yamaguchi, Y. Ohkoshi, Y. Goth, and M. Nagura, Seikei-Kakou, 17, 649 (2005).

17. “Gomu Erastmer Katsuyoh Note”, Gomu Erasmer Kenkyukai, p. 165, Kogyo Chosakai Publishing, Inc (1990).

18. S. Kase and T. Matsuo, J. Polym. Sci. A, 3, 2541 (1965). 